Received: 30.04 .2020

Revised: 01.06.2020

Accepted: 19.06 .2020

DOI: $10.17804 / 2410-9908.2020 .3 .029-046$

\title{
ISOTHERMAL LAYERED FLOWS OF A VISCOUS INCOMPRESSIBLE FLUID WITH SPATIAL ACCELERATION IN THE CASE OF THREE CORIOLIS PARAMETERS
}

\author{
N. V. Burmasheva ${ }^{a} *$, E. Yu. Prosviryakov ${ }^{\text {b) }}$ \\ Institute of Engineering Science, Ural Branch of the Russian Academy of Sciences, \\ 34 Komsomolskaya St., Ekaterinburg, 620049, Russian Federation \\ a) (iD https://orcid.org/0000-0003-4711-1894 nat_burm@mail.ru; \\ b) (iD https://orcid.org/0000-0002-2349-7801 《evgen_pros@mail.ru; \\ *Corresponding author. E-mail: nat_burm@mail.ru \\ Address for correspondence: 34 Komsomolskaya St., Ekaterinburg, 620049, Russian Federation \\ Tel.: +7 (343) 37535 76; fax: +7 (343) 3745330
}

We study the solvability of the overdetermined system of Navier-Stokes equations, supplemented by the incompressibility equation, which is used to describe isothermal large-scale shear flows of a rotating viscous incompressible fluid. Large-scale flows are studied in a thin-layer approximation (the vertical velocity of the fluid is assumed to be zero). The rotation of a continuous fluid medium is described by three Coriolis parameters. The solution of the reduced system of Navier-Stokes equations is constructed in the Lin-Sidorov-Aristov class. In this case, both nonzero components of the velocity vector, the pressure and temperature fields are assumed to be full linear forms of two Cartesian coordinates, and the dependence on the third Cartesian coordinate has an arbitrary form (including non-polynomial). It is shown that the nonlinear overdetermined system of Navier-Stokes equations and of the incompressibility equation in the framework of the LinSidorov-Aristov class reduces to the equivalent nonlinear overdetermined system of ordinary differential equations, in which the components of the hydrodynamic fields act as unknown functions. The compatibility condition for the equations of the resulting system is derived. It is shown that, if this compatibility condition is fulfilled, the system has a unique solution, and the spatial accelerations in both variables (the linearity with respect to them was postulated when choosing the solution class) prove to be constant functions. These results are a generalization of similar results obtained earlier in the study of solvability in the cases of one and two Coriolis parameters.

Keywords: exact solution, shear flow, Coriolis parameter, solvability, Navier-Stokes equation, overdetermined system, large-scale flow.

\section{References}

1. Monin A.S. Theoretical Foundations of Geophysical Hydrodynamics. Leningrad, Gidrometeoizdat Publ., 1988. (In Russian).

2. Brekhovskikh L., Goncharov V. Mechanics of Continua and Wave Dynamics, transl. from Rusian, Springer-Verlag Berlin Heidelberg, 1985. DOI: 10.1007/978-3-642-96861-7.

3. Zyryanov V.N. Theory of steady ocean currents, Leningrad, Gidrometeoizdat Publ., 1985. (in Russian).

4. Pedlosky J. Geophysical fluid dynamics, Berlin, New York, Springer-Verlag, 1987.

5. Gill A.E. Atmosphere-ocean dynamics, Cambridge, University of Cambridge, 1982.

6. Ekman V.W. On the influence of the Earth's rotation on ocean-currents. Ark. Mat. Astron. Fys., 1905, vol. 2, no. 11, pp. 1-52. 
7. Gershuni G.Z., Zhukhovitskii E.M. Convective Stability of Incompressible Fluids, Jerusalem, Keter Publishing House, 1976.

8. $\quad$ Landau L.D., Lifshitz E.M. Course of Theoretical Physics. Vol. 6. Fluid mechanics, Oxford, Pergamon Press, 1987.

9. Temam R. Navier-Stokes Equations: Theory and Numerical Analysis, Amsterdam, New York, Oxford, North-Holland Publ., 1977.

10. Polyanin A.D., Kutepov A.M., Kazenin D.A., Vyazmin A.V. Hydrodynamics, Mass and Heat Transfer in Chemical Engineering, Boca Raton, CRC Press, 2001.

11. Couette M. Etudes sur le frottement des liquids. Ann. Chim. Phys., 1890, vol. 21, pp. 433-510.

12. Guermond, J.L., Migeon C., Pineau G., Quartapel L. Start-up flows in a three-dimensional rectangular driven cavity of aspect ratio 1:1:2 at $R e=1000$. J. Fluid Mech., 2002, vol. 450, pp. 169-199. DOI: 10.1017/S0022112001006383.

13. Neto C., Evans D., Bonaccurso E., Butt H.-J., Craig V.S.J. Boundary slip in Newtonian liquids: a review of experimental studies. Reports on Progress in Physics, 2005, vol. 68, pp. 2859-2897. DOI: $10.1088 / 0034-4885 / 68 / 12 / R 05$.

14. Aristov S.N., Frik P.G. Nonlinear effects of the Ekman layer on the dynamics of large-scale eddies in shallow water. Journal of Applied Mechanics and Technical Physics, 1991, vol. 32, no. 2, pp. 189-194. DOI: 10.1007/BF00858033.

15. Aristov S.N., Shvarts K.G. Vortical Flows of the Advective Nature in a Rotating Fluid Layer, Perm, Perm State Univ. Publ., 2006. (In Russian).

16. Aristov S.N., Shvarts K.G. Vikhrevye techeniia v tonkikh sloiakh zhidkosti [Vortical Flows in Thin Fluid Layers]. Kirov, Vyatka State Univ. Publ., 2011. (In Russian).

17. Gorshkov A.V., Prosviryakov E.Y. Ekman convective layer flow of a viscous incompressible fluid. Izvestiya. Atmospheric and Oceanic Physics, 2018, vol. 54, no 2, pp. 189-195. DOI: $10.1134 /$ S0001433818020081.

18. Kompaniets L., Pitalskaya O. Exact solutions of Ekmans model for three-dimensional windinduced flow of homogeneous fluid with geostrophic current. Computer Research and Modeling, 2009, vol. 1, pp. 57-66. DOI: 10.20537/2076-7633-2009-1-1-57-66. (In Russian).

19. Aristov S.N., Knyazev D.V., Polyanin A.D. Exact solutions of the Navier-Stokes Equations with the linear dependence of velocity components on two space variables. Theoretical Foundations of Chemical Engineering, 2009, vol. 43, no. 5, pp. 642-662. DOI: 10.1134/S0040579509050066.

20. Shrira V.I., Almelah R.B. Upper-ocean Ekman current dynamics: a new perspective. Journal of Fluid Mechanics, 2020, vol. 887, A24. DOI: 10.1017/jfm.2019.1059.

21. Fečkan M., Guan Y., O'Regan D., Wang J.R. Existence and uniqueness and first order approximation of solutions to atmospheric Ekman flows. Monatshefte für Mathematik, 2020. DOI: $10.1007 / \mathrm{s} 00605-020-01414-7$.

22. Ortiz-Tarin J.L., Lee S., Flores O., Sarkar S. Global modes and large-scale structures in an Ekman boundary layer. Journal of Physics: Conference Series, 2020, vol. 1522, 012011. DOI: $10.1088 / 1742-6596 / 1522 / 1 / 012011$.

23. Constantin A., Johnson R.S. Atmospheric Ekman flows with variable eddy viscosity. Boundary-Layer Meteorol, 2019, vol. 170, pp. 395-414. DOI: 10.1007/s10546-018-0404-0.

24. Prosviryakov E.Y. New class of exact solutions of Navier-Stokes equations with exponential dependence of velocity on two spatial coordinates. Theoretical Foundations of Chemical Engineering, 2019, vol. 53, no. 1, pp. 107-114. DOI: 10.1134/S0040579518060088.

25. Burmasheva N.V., Prosviryakov E.Yu. Exact solution of Navier-Stokes equations describing spatially inhomogeneous flows of a rotating fluid. Trudy Instituta Matematiki i Mekhaniki UrO RAN, 2020, vol. 26, no. 2., pp. 79-87. (In Russian).

26. Burmasheva N.V., Prosviryakov E.Yu. A class of exact solutions for two-dimensional equations of geophysical hydrodynamics with two Coriolis parameters. The Bulletin of Irkutsk State 
University. Series Mathematics, 2020, vol. 32, pp. 33-48. DOI: 10.26516/1997-7670.2020.32.33. (In Russian).

27. Lin C.C. Note on a class of exact solutions in magneto-hydrodynamics. Arch. Rational Mech. Anal., 1958, vol. 1, pp. 391-395.

28. Aristov S.N., Prosviryakov E.Yu. Large-scale flows of viscous incompressible vortical fluid. Russian Aeronautics, 2015, vol. 58, no. 4, pp. 413-418. DOI: 10.3103/S1068799815040091.

29. Aristov S.N., Prosviryakov E.Y. Inhomogeneous Couette flow. Russian Journal of Nonlinear Dynamics, 2014, vol. 10, no. 2, pp. 177-182. DOI: 10.20537/nd1402004. (In Russian).

30. Prosviryakov E.Y. Exact Solutions for three-dimensional potential and vorticity Couette flows of an incompressible viscous fluid. Vestnik Natsional'nogo issledovatel'skogo yadernogo universiteta "MIFI", 2015, vol. 4, no. 6, pp. 501-506 DOI: 10.1134/S2304487X15060127. (In Russian).

31. Aristov S.N., Prosviryakov E.Y. Unsteady layered vortical fluid flows. Fluid Dynamics, 2016, vol. 51, no. 2, pp. 148-154. DOI: 10.1134/S0015462816020034.

32. Aristov S.N., Prosviryakov E.Y. A new class of exact solutions for three-dimensional thermal diffusion equations. Theoretical Foundations of Chemical Engineering, 2016, vol. 50, no. 3, pp. 286-293. DOI: 10.1134/S0040579516030027.

33. Burmasheva N.V., Prosviryakov E.Yu. A large-scale layered stationary convection of an incompressible viscous fluid under the action of shear stresses at the upper boundary. Velocity field investigation. VESTNIK SAMARSKOGO GOSUDARSTVENNOGO TEKHNICHESKOGO UNIVERSITETA-SERIYA-FIZIKO-MATEMATICHESKIYE NAUKI, 2017, vol. 21, no. 1, pp. 180-196. DOI: 10.14498/vsgtu1527. (In Russian).

34. Burmasheva N.V., Prosviryakov E.Yu. A large-scale layered stationary convection of a incompressible viscous fluid under the action of shear stresses at the upper boundary. Temperature and presure field investigation. VESTNIK SAMARSKOGO GOSUDARSTVENNOGO TEKHNICHESKOGO UNIVERSITETA-SERIYA-FIZIKO-MATEMATICHESKIYE NAUKI, 2017, vol. 21, no. 4, pp. 736-751 DOI: 10.14498/vsgtu1568. (In Russian).

35. Gorshkov A.V., Prosviryakov E.Yu. Analytic solutions of stationary complex convection describing a shear stress field of different signs. Trudy Inst. Mat. i Mekh. UrO RAN, 2017, vol. 23, no. 2, pp. 32-41. DOI: 10.21538/0134-4889-2017-23-2-32-41. (In Russian).

36. Vereshchagin V.P., Subbotin Yu.N., Chernykh N.I. Description of a helical motion of an incompressible nonviscous fluid. Proc. Steklov Inst. Math., 2015, vol. 288, pp. 202-210. DOI: 10.1134/S0081543815020212.

37. Vereshchagin V.P., Subbotin Yu.N., Chernykh N.I. Some solutions of continuum equations for an incompressible viscous medium. Proc. Steklov Inst. Math., 2014, vol. 287, pp. 208-223. DOI: $10.1134 /$ S008154381409020X.

38. Vereshchagin V.P., Subbotin Yu.N., Chernykh N.I. On the mechanics of helical flows in an ideal incompressible nonviscous continuous medium. Proc. Steklov Inst. Math., 2014, vol. 284, pp. 159-174. DOI: 10.1134/S008154381402014X.

39. Zubarev N.M., Prosviryakov E.Yu. Exact solutions for the layered three-dimensional nonstationary isobaric flows of viscous incompressible fluid. Journal of Applied Mechanics and Technical Physics, 2019, vol. 60, no. 6 (358), pp. 65-71. DOI: 10.15372/PMTF201. (In Russian).

40. Pukhnachev V.V. Point vortex in a viscous incompressible fluid. Journal of Applied Mechanics and Technical Physics, 2014, vol. 55, no. 2, pp. 345-351. DOI: 10.1134/S0021894414020175.

41. Birikh R.V., Pukhnachev V.V., Frolovskaya O.A. Convective flow in a horizontal channel with non-Newtonian surface rheology under time-dependent longitudinal temperature gradient. Fluid Dynamics, 2015, vol. 50, no. 1, pp. 173-179. DOI: 10.1134/S0015462815010172.

42. Golubkin V.N., Sizykh G.B. Maximum principle for the Bernoulli function. TsAGI Science Journal, 2015, vol. 46, no. 5, pp. 485-490. DOI: 10.1615/TsAGISciJ.v46.i5.50. 
43. Sizykh G.B. Axisymmetric helical flows of viscous fluid. Russian Mathematics, 2019, vol. 63, no. 2, pp. 44-50. DOI: 10.3103/S1066369X19020063.

44. Sizykh G.B. The splitting of Navier-Stokes equations for a class of axisymmetric flows. VESTNIK SAMARSKOGO GOSUDARSTVENNOGO TEKHNICHESKOGO UNIVERSITETASERIYA-FIZIKO-MATEMATICHESKIYE NAUKI, 2020, vol. 24, no. 1, pp. 163-173. DOI: 10.14498/vsgtu1740. (In Russian).

45. Markov V.V., Sizykh G.B. Exact solutions of the Euler equations for some twodimensional incompressible flows. Proc. Steklov Inst. Math., 2016, vol. 294, no. 1, pp. 283-290. DOI: $10.1134 / \mathrm{S} 0081543816060195$.

46. Ershkov S.V., Shamin R.V., Giniyatullina A.R. On a new type of non-stationary helical flows for incompressible 3D Navier-Stokes equations. Journal of King Saud University - Science, 2020, vol. 32, no. 1, pp. 459-467. DOI: 10.1016/j.jksus.2018.07.006.

47. Kovalev V.P., Prosviryakov E.Yu., Sizykh G.B. Obtaining examples of exact solutions of the Navier-Stokes equations for helical flows by the method of summation of velocities. Trudy MFTI, 2017, vol. 9, no. 1, pp. 71-88. (In Russian).

48. Burmasheva N.V., Prosviryakov E.Yu. Thermocapillary convection of a vertical swirling liquid. Theoretical Foundations of Chemical Engineering, 2020, vol. 54, no. 1, pp. 230-239. DOI: $10.1134 / \mathrm{S} 0040579519060034$.

49. Ul'yanov O. N. A class of viscous fluid flows. Proc. Steklov Inst. Math. (Suppl.), 2003, suppl. 2, S173-S181.

50. Pukhnachev V.V. Nonlinear diffusion and exact solutions to the Navier-Stokes equations. The Bulletin of Irkutsk State University. Series Mathematics, 2010, vol. 3, no. 1, pp. 61-69.

51. Burmasheva N.V., Prosviryakov E.Yu. Convective layered flows of a vertically whirling viscous incompressible fluid. Velocity field investigation. VESTNIK SAMARSKOGO GOSUDARSTVENNOGO TEKHNICHESKOGO UNIVERSITETA-SERIYA-FIZIKO-MATEMATICHESKIYE NAUKI， 2019, vol. 23, no. 2, pp. 341-360. DOI: 10.14498/vsgtu1670.

52. Privalova V.V., Prosviryakov E.Yu., Simonov M.A. Nonlinear gradient flow of a vertical vortex fluid in a thin layer. Russian Journal of Nonlinear Dynamics, 2019, vol. 15, no. 3, pp. 271-283. DOI: $10.20537 /$ nd190306.

53. Aristov S.N., Privalova V.V., Prosviryakov E.Yu. Stationary nonisothermal Couette flow. Quadratic heating of the upper boundary of the fluid layer. Nelin. Dinam., 2016, vol. 12, no. 2, pp. 167-178. (In Russian).

54. Polyanin A.D., Zaitsev V.F. Handbook of exact solutions for ordinary differential equations, 2nd ed., Boca Raton: Chapman\& Hall/CRC, 2003. 
Подана в журнал: 30.04 .2020

УдК 517.958

DOI: $10.17804 / 2410-9908.2020 .3 .029-046$

\title{
ИЗОТЕРМИЧЕСКИЕ СДВИГОВЫЕ ТЕЧЕНИЯ ВЯЗКОЙ НЕСЖИМАЕМОЙ ЖИДКОСТИ С ПРОСТРАНСТВЕННЫМ УСКОРЕНИЕМ ПРИ УЧЕТЕ ТРЕХ ПАРАМЕТРОВ КОРИОЛИСА
}

\author{
Н. В. Бурмашева ${ }^{\text {a)*, Е. Ю. Просвиряков }}{ }^{\text {क) }}$ \\ Институт машиноведения УрО РАН, \\ 34, ул. Комсомольская, г. Екатеринбург, Российская Федераџия \\ a) (iD https://orcid.org/0000-0003-4711-1894 nat_burm@mail.ru; \\ б) (iD https://orcid.org/0000-0002-2349-7801 《evgen_pros@mail.ru; \\ *Ответственный автор. Электронная почта: nat_burm@mail.ru \\ Адрес для переписки: ул. Комсомольская, 34, г. Екатеринбург, Российская Федерация \\ Тел.: +7 (343) 375-35-76; факс: 374-53-30
}

Исследуется вопрос разрешимости переопределенной системы уравнений НавьеСтокса, дополненной уравнением несжимаемости, используемой для описания изотермических крупномасштабных сдвиговых течений вращающейся вязкой несжимаемой жидкости. Крупномасштабные течения изучаются в приближении тонкого слоя (вертикальная скорость жидкости полагается равной нулю). Вращение сплошной жидкой среды описывается тремя параметрами Кориолиса. Решение редуцированной системы уравнений Навье-Стокса строится в классе Линя-Сидорова-Аристова. При этом обе ненулевые компоненты вектора скорости, поля давления и температуры полагаются полными линейными формами двух декартовых координат, а зависимость от третьей декартовой координаты имеет произвольный вид (в том числе и неполиномиальный). Нелинейная переопределенная система уравнений Навье-Стокса и уравнения несжимаемости в рамках класса Линя-Сидорова-Аристова сводится к эквивалентной нелинейной переопределенной системе обыкновенных дифференциальных уравнений, в которой в качестве неизвестных функций выступают компоненты гидродинамических полей. Выведено условие совместности уравнений полученной системы. Показано, что если условие совместности выполняется, то система имеет единственное решение, причем пространственные ускорения по обеим переменным, линейность относительно которых постулировалась при выборе класса решений, оказываются постоянными функциями. Результаты являются обобщением аналогичных результатов, полученных ранее при исследовании разрешимости в случаях одного и двух параметров Кориолиса.

Ключевые слова: точное решение, сдвиговое течение, параметр Кориолиса, разрешимость, уравнение Навье-Стокса, переопределенная система, крупномасштабное течение.

\section{1. Введение}

При описании движения вращающихся жидкостей традиционно используется приближение, согласно которому сила инерции характеризуется только одним параметром Кориолиса [1-5]. Этого подхода придерживался Экман [6] при описании вращающегося океана бесконечной глубины. Одним из обоснований служили характерные значения горизонтальных скоростей в геофизической гидродинамике значительно превышают соответствующие значения вертикальной проекции вектора скорости течения [1,3-5]. Классическое крупномасштабное течение Экмана является изотермическим. Горизонтальные проекции поля скорости определялись из системы уравнений Навье-Стокса [7-10], дополненной уравнением несжимаемости. Экман, полагая скорости относящимися к классу течений типа Куэтта [11], построил нетривиальное решение описанной системы. 
Однако предположение об однородности распределения компонент поля скорости не всегда отвечает данным натурных экспериментов [12, 13].

Точное решение Экмана многократно обобщалось [1, 4, 14-24]. Достаточно часто при этом возникали ситуации, в которых определяющая система уравнений оказывалась переопределенной. Было построено решение для скоростей наиболее общего вида в рамках класса Линя-Сидорова-Аристова при учете одного ненулевого параметра Кориолиса в работе [25]. При удовлетворении определенному алгебраическому условию переопределенная редуцированная система уравнений движения вращающейся жидкости оказывается разрешимой. Аналогичное условие было получено в работе [26], но для случая, когда сила инерции описывается двумя ненулевыми параметрами Кориолиса. В работе предпринята попытка интегрирования системы уравнений Навье-Стокса в случае, когда все три проекции вектора угловой скорости вращения отличны от нуля.

\section{2. Постановка задачи}

Рассмотрим локальную (неинерционную) систему координат $O x y z$, соответствующую рассматриваемой области течения жидкости. Пусть эта система вращается вокруг некоторой оси с угловой скоростью $\Omega=1 / 2\left(f_{3}, f_{2}, f_{1}\right)$. Параметры $f_{1}=2 \Omega \cos \varphi_{1}, f_{2}=2 \Omega \cos \varphi_{2}$, $f_{3}=2 \Omega \cos \varphi_{3}$ называются параметрами Кориолиса, где $\Omega$ - величина угловой скорости вращения, $\varphi_{1} ; \varphi_{2} ; \varphi_{3}$ - углы между вектором $\boldsymbol{\Omega}$ и положительными направлениями координатных осей $O x, O y, O z$ соответственно [1-5]. В качестве такой области может выступать, например, некоторая часть Мирового океана, а в качестве оси вращения - Земная ось. Будем полагать, что компоненты $f_{1}, f_{2}, f_{3}$ вектора угловой скорости не зависят от времени. Это допущение оправдано тем, что несмотря на влияние колебания оси вращения Земли на динамику крупномасштабных течений Мирового океана, оно оказывается достаточно мало, чтобы можно было им пренебречь [3].

Введем в рассмотрение редуцированное давление

$$
P=[p-\rho((\boldsymbol{\Omega} \times \boldsymbol{r}),(\boldsymbol{\Omega} \times \boldsymbol{r})) / 2-\rho g] / \rho=p / \rho-((\boldsymbol{\Omega} \times \boldsymbol{r}),(\boldsymbol{\Omega} \times \boldsymbol{r})) / 2-g,
$$

вычтя из реального давления $p$ центробежную составляющую $\rho((\boldsymbol{\Omega} \times \boldsymbol{r}),(\boldsymbol{\Omega} \times \boldsymbol{r})) / 2$ и силу тяжести и разделив получившуюся разность на плотность жидкости $\rho$. В этом случае уравнение Навье-Стокса (уравнение сохранения количества) движения и уравнение несжимаемости принимает вид [3]

$$
\frac{\partial \boldsymbol{V}}{\partial t}+(\boldsymbol{V}, \nabla) \boldsymbol{V}+2 \boldsymbol{\Omega} \times \boldsymbol{V}=-\nabla P+v \Delta \boldsymbol{V}, \nabla \cdot \boldsymbol{V}=0
$$

В уравнении (1) приняты обозначения: $\boldsymbol{V}=\left(V_{x}, V_{y}, V_{z}\right)$ - вектор скорости движения, $v$ - кинематическая вязкость жидкости; $\nabla=(\partial / \partial x, \partial / \partial y, \partial / \partial z)$ - оператор Гамильтона, $\Delta=\partial^{2} / \partial x^{2}+\partial^{2} / \partial y^{2}+\partial^{2} / \partial z^{2}-$ оператор Лапласа. Отметим, что ускорение Кориолиса $2 \boldsymbol{\Omega} \times \boldsymbol{V}$ в системе уравнений (1) зависит от величины проекций вектора угловой скорости $\Omega$ на координатные оси: 


$$
2 \boldsymbol{\Omega} \times \boldsymbol{V}=\left|\begin{array}{ccc}
\boldsymbol{i} & \boldsymbol{j} & \boldsymbol{k} \\
f_{3} & f_{2} & f_{1} \\
V_{x} & V_{y} & V_{z}
\end{array}\right|=\left(f_{2} V_{z}-f_{1} V_{y}, f_{1} V_{x}-f_{3} V_{z}, f_{3} V_{y}-f_{2} V_{x}\right)
$$

В случае установившихся крупномасштабных сдвиговых $\left(V_{z}=0\right)$ течений проекции уравнения (1) на координатные оси запишем в следующем виде:

$$
\begin{gathered}
\left(V_{x} \frac{\partial}{\partial x}+V_{y} \frac{\partial}{\partial y}\right) V_{x}-f_{1} V_{y}=-\frac{\partial P}{\partial x}+v\left(\frac{\partial^{2}}{\partial x^{2}}+\frac{\partial^{2}}{\partial y^{2}}+\frac{\partial^{2}}{\partial z^{2}}\right) V_{x} \\
\left(V_{x} \frac{\partial}{\partial x}+V_{y} \frac{\partial}{\partial y}\right) V_{y}+f_{1} V_{x}=-\frac{\partial P}{\partial y}+v\left(\frac{\partial^{2}}{\partial x^{2}}+\frac{\partial^{2}}{\partial y^{2}}+\frac{\partial^{2}}{\partial z^{2}}\right) V_{y} \\
f_{3} V_{y}-f_{2} V_{x}=-\frac{\partial P}{\partial z}, \frac{\partial V_{x}}{\partial x}+\frac{\partial V_{y}}{\partial y}=0 .
\end{gathered}
$$

Система (2)-(4) является переопределенной, поскольку для нахождения двух скоростей и модифицированного давления имеется четыре уравнения. Разрешимость системы уравнений будем исследовать в классе точных решений Линя-Сидорова-Аристова.

Рассмотрим далее следующий класс точных решений [25-32]:

$$
\begin{gathered}
V_{x}=U(z)+u_{1}(z) x+u_{2}(z) y, V_{y}=V(z)+v_{1}(z) x+v_{2}(z) y ; \\
P=P_{0}(z)+P_{1}(z) x+P_{2}(z) y+P_{12}(z) x y+P_{11}(z) \frac{x^{2}}{2}+P_{22}(z) \frac{y^{2}}{2} .
\end{gathered}
$$

Подставим класс (5) в систему (2)-(4). В результате получим следующие соотношения:

$$
\begin{aligned}
& \left(\left(U+u_{1} x+u_{2} y\right) \frac{\partial}{\partial x}+\left(V+v_{1} x+v_{2} y\right) \frac{\partial}{\partial y}\right)\left(U+u_{1} x+u_{2} y\right)-f_{1}\left(V+v_{1} x+v_{2} y\right)= \\
= & -\frac{\partial\left(P_{0}+P_{1} x+P_{2} y+P_{12} x y+P_{11} \frac{x^{2}}{2}+P_{22} \frac{y^{2}}{2}\right)}{\partial x}+v\left(\frac{\partial^{2}}{\partial x^{2}}+\frac{\partial^{2}}{\partial y^{2}}+\frac{\partial^{2}}{\partial z^{2}}\right)\left(U+u_{1} x+u_{2} y\right) ; \\
& \left(\left(U+u_{1} x+u_{2} y\right) \frac{\partial}{\partial x}+\left(V+v_{1} x+v_{2} y\right) \frac{\partial}{\partial y}\right)\left(V+v_{1} x+v_{2} y\right)+f_{1}\left(U+u_{1} x+u_{2} y\right)= \\
& -\frac{\partial\left(P_{0}+P_{1} x+P_{2} y+P_{12} x y+P_{11} \frac{x^{2}}{2}+P_{22} \frac{y^{2}}{2}\right)}{\partial y}+v\left(\frac{\partial^{2}}{\partial x^{2}}+\frac{\partial^{2}}{\partial y^{2}}+\frac{\partial^{2}}{\partial z^{2}}\right)\left(V+v_{1} x+v_{2} y\right) ;
\end{aligned}
$$




$$
f_{3}\left(V+v_{1} x+v_{2} y\right)-f_{2}\left(U+u_{1} x+u_{2} y\right)=-\frac{\partial\left(P_{0}+P_{1} x+P_{2} y+P_{12} x y+P_{11} \frac{x^{2}}{2}+P_{22} \frac{y^{2}}{2}\right)}{\partial z} .
$$

Вычислим соответствующие частные производные в уравнениях (6)-(8), в результате чего уравнения примут более простой вид:

$$
\begin{gathered}
\left(U+u_{1} x+u_{2} y\right) u_{1}+\left(V+v_{1} x+v_{2} y\right) u_{2}-f_{1}\left(V+v_{1} x+v_{2} y\right)= \\
=-\left(P_{1}+P_{12} y+P_{11} x\right)+v\left(U^{\prime \prime}+u_{1}{ }^{\prime} x+u_{2}{ }^{\prime \prime} y\right) \\
\left(U+u_{1} x+u_{2} y\right) v_{1}+\left(V+v_{1} x+v_{2} y\right) v_{2}+f_{1}\left(U+u_{1} x+u_{2} y\right)= \\
=-\left(P_{2}+P_{12} x+P_{22} y\right)+v\left(V^{\prime \prime}+v_{1}{ }^{\prime \prime} x+v_{2}{ }^{\prime \prime} y\right) ; \\
f_{3}\left(V+v_{1} x+v_{2} y\right)-f_{2}\left(U+u_{1} x+u_{2} y\right)=-\left(P_{0}{ }^{\prime}+P_{1}^{\prime} x+P_{2}^{\prime} y+P_{12}{ }^{\prime} x y+P_{11}{ }^{\prime} \frac{x^{2}}{2}+P_{22}{ }^{\prime} \frac{y^{2}}{2}\right) .
\end{gathered}
$$

Воспользуемся далее принципом неопределенных коэффициентов и приравняем в уравнениях (9)-(11) выражения при независимых переменных $x, y$. Получим систему нелинейных обыкновенных дифференциальных уравнений, производная в которых берется по переменной $z$. Сгруппируем эти уравнения следующим образом:

$$
\begin{gathered}
P_{11}{ }^{\prime}=0, P_{12}{ }^{\prime}=0, P_{22}{ }^{\prime}=0 \\
v u_{1}{ }^{\prime}=u_{1}^{2}+\left(u_{2}-f_{1}\right) v_{1}+P_{11} ; \\
v u_{2}{ }^{\prime}=u_{1} u_{2}+\left(u_{2}-f_{1}\right) v_{2}+P_{12} ; \\
v v_{1}^{\prime \prime}=u_{1}\left(v_{1}+f_{1}\right)+v_{1} v_{2}+P_{12} ; \\
v v_{2}{ }^{\prime}=u_{2}\left(v_{1}+f_{1}\right)+v_{2}^{2}+P_{22} ; \\
P_{1}^{\prime}=f_{2} u_{1}-f_{3} v_{1} ; \\
P_{2}{ }^{\prime}=f_{2} u_{2}-f_{3} v_{2} ; \\
v U^{\prime \prime}=U u_{1}+V\left(u_{2}-f_{1}\right)+P_{1} ; \\
v V^{\prime \prime}=U\left(v_{1}+f_{1}\right)+V v_{2}+P_{2} ;
\end{gathered}
$$




$$
P_{0}{ }^{\prime}=f_{2} U-f_{3} V
$$

Подставим аналогичным образом линейные формы (5) в уравнение несжимаемости:

$$
\nabla \cdot \boldsymbol{V}=0
$$

После вычисления соответствующих частных производных придем к следующему условию:

$$
u_{1}+v_{2}=0
$$

Перепишем далее систему уравнений (12)-(16) с учетом соотношения (17), в результате элементарных алгебраических преобразований получим:

$$
\begin{gathered}
P_{11}{ }^{\prime}=0 ; P_{12}{ }^{\prime}=0 ;, P_{22}{ }^{\prime}=0 ; \\
v u_{1}{ }^{\prime}=u_{1}{ }^{2}+\left(u_{2}-f_{1}\right) v_{1}+P_{11} ; \\
v u_{2}{ }^{\prime \prime}=f_{1} u_{1}+P_{12} ; \\
v v_{1}{ }^{\prime}=f_{1} u_{1}+P_{12} ; \\
-v u_{1}{ }^{\prime \prime}=u_{1}{ }^{2}+u_{2}\left(v_{1}+f_{1}\right)+P_{22} ; \\
P_{1}^{\prime}=f_{2} u_{1}-f_{3} v_{1} ; \\
P_{2}^{\prime}=f_{2} u_{2}-f_{3}\left(-u_{1}\right)=f_{3} u_{1}+f_{2} u_{2} ; \\
v U^{\prime \prime}=U u_{1}+V\left(u_{2}-f_{1}\right)+P_{1} ; \\
v V^{\prime \prime}=U\left(v_{1}+f_{1}\right)-V u_{1}+P_{2} ; \\
P_{0}{ }^{\prime}=f_{2} U-f_{3} V .
\end{gathered}
$$

Из уравнения (18) непосредственно следует, что коэффициенты при квадратичных слагаемых поля давления (5) являются постоянными величинами:

$$
P_{i j}=\operatorname{const}(i, j=1,2) \text {. }
$$

Оставшиеся уравнения (подсистемы (19)-(22)) образуют переопределенную нелинейную систему обыкновенных дифференциальных уравнений, поскольку число соотношений в ней превосходит число неизвестных функций. Причем, если в подсистемах (20)-(22) число уравнений совпадает с числом неизвестных, то в подсистеме (19) число неизвестных меньше 
числа уравнений. Таким образом, если удастся найти совместное решение уравнений (19), то система (18)-(22) перестанет быть переопределенной.

Заметим, что проблема переопределенности подсистемы (19) имеет место, если только рассматривается полный класс вида (5), т. е. когда обе ненулевые компоненты вектора скорости линейны одновременно по двум горизонтальным координатам. В частном случае класса (5), когда вектор скорости описывается только однородными компонентами

$$
V_{x}=U, V_{y}=V
$$

подсистема (19) сводится к условиям $P_{i j}=0(i, j=1,2)$, что не противоречит подсистеме (18). Структура поля скоростей вида (23) рассмотрена, например, в работах [33-41]. При этом поле скоростей вида (23) может описывать только вертикально незавихренные течения вязкой несжимаемой жидкости.

Для описания вертикально завихренных жидкостей можно использовать другой частный случай класса (5) - случай, когда поле скоростей имеет, например, следующий вид:

$$
V_{x}=U+u_{2} y, V_{y}=V .
$$

Течения вязкой жидкости в рамках класса вида (24) начали изучаться в работах [42-53].

\section{3. Анализ переопределенной подсистемы}

Решение уравнений (19) не зависит от второго и третьего параметров Кориолиса. Точно такая же система для определения пространственных ускорений $u_{1}, u_{2}, v_{1}, v_{2}$ при постоянных квадратичных неоднородностях $P_{i j}$ давления жидкости была получена и подробно исследована [25,26]. В работах [25,26] было показано, что система (19) разрешима, если существует вещественное число $c$, являющееся корнем следующего квадратного уравнения:

$$
c^{2} f_{1}^{2}+2 c f_{1}^{3}-P_{11}^{2}-4 P_{12}^{2}-2 f_{1}^{2}\left(P_{11}-P_{22}\right)+2 P_{11} P_{22}+P_{22}^{2}=0 \text {. }
$$

В этом случае пространственные ускорения $u_{1}, u_{2}, v_{1}, v_{2}$ описываются точным решением, которое не зависит от поперечной координаты $z$ :

$$
u_{1}=-\frac{P_{12}}{f_{1}} ; u_{2}=\frac{P_{11}-P_{22}-f_{1} c}{2 f_{1}} ; v_{1}=\frac{P_{11}-P_{22}+f_{1} c}{2 f_{1}} ; v_{2}=\frac{P_{12}}{f_{1}}
$$

В зависимости от значения дискриминанта квадратного уравнения (25), определяющего значение параметра $c$, выражения (26) могут описывать как единственное решение, так и серию из двух решений.

Далее определим вид оставшихся коэффициентов форм (5). Для этого необходимо проинтегрировать систему уравнений (20)-(22). Начнем с системы (20). С учетом выражений (25) правая часть обоих уравнений подсистемы (20) является постоянной функцией. При однократном интегрировании такой функции по переменной $z$ получим линейную функцию этой переменной:

$$
P_{1}=\alpha_{1} z+p_{1} ; P_{2}=\alpha_{2} z+p_{2}
$$


где $\alpha_{1}=f_{2} u_{1}-f_{3} v_{1} ; \alpha_{2}=f_{3} u_{1}+f_{2} u_{2} ;$ a $p_{1}$ ѐ $p_{2}-$ постоянные интегрирования. Зная точные решения (27) для градиентов $P_{1}$ и $P_{2}$ поля давления (5), можем найти однородные слагаемые $U$ и $V$ поля скорости. Для этого проанализируем структуру системы (21).

Уравнения (21) есть система обыкновенных дифференциальных неоднородных уравнений четвертого порядка с постоянными коэффициентами и известной линейной неоднородностью. В общем случае уравнения (21) связаны друг с другом, т.е. нельзя проинтегрировать одно из уравнений этой системы независимо от другого уравнения. Однако при некоторых значениях коэффициентов $u_{1}, u_{2}, v_{1}$, по крайней мере, одно из этих уравнений может становиться изолированным. Другими словами, вид решения системы (21) зависит от величины этих параметров. Рассмотрим возможные случаи.

1. Пусть $v_{1}+f_{1}=0$, тогда второе уравнение системы (21) примет более простой вид:

$$
v V^{\prime \prime}+u_{1} V=P_{2}
$$

В уравнении (28) в роли неизвестного выступает только скорость $V$, следовательно, решение этого уравнения может быть найдено независимо от значения скорости $U$. Компонента $U$ определяется из первого уравнения системы (21). При этом вид решения уравнения (28) зависит от значения пространственного ускорения $u_{1}$.

1.1. Если $u_{1}=0$, то однородные компоненты $U$ и $V$ скоростей $V_{x}$ и $V_{y}$ соответственно и фоновое давление $P_{0}$ описываются полиномиальными формулами:

$$
\begin{gathered}
V=\frac{\alpha_{2}}{6 v} z^{3}+\frac{p_{2}}{2 v} z^{2}+C_{2} z+C_{1} \\
U=\frac{\left(u_{2}-f_{1}\right) \alpha_{2}}{120 v^{2}} z^{5}+\frac{p_{2}\left(u_{2}-f_{1}\right)}{24 v^{2}} z^{4}+\frac{\left(C_{2}\left(u_{2}-f_{1}\right)+\alpha_{1}\right)}{6 v} z^{3}+\frac{\left(C_{1}\left(u_{2}-f_{1}\right)+p_{1}\right)}{2 v} z^{2}+C_{4} z+C_{3} ; \\
P_{0}=\frac{f_{2} \alpha_{2}\left(u_{2}-f_{1}\right)}{720 v^{2}} z^{6}+\frac{f_{2} p_{2}\left(u_{2}-f_{1}\right)}{120 v^{2}} z^{5}+\frac{f_{2}\left(C_{2}\left(u_{2}-f_{1}\right)+\alpha_{1}\right)-f_{3} \alpha_{2}}{24 v} z^{4}+ \\
+\frac{f_{2}\left(C_{1}\left(u_{2}-f_{1}\right)+p_{1}\right)-f_{3} p_{2}}{6 v} z^{3}+\frac{C_{4} f_{2}-C_{2} f_{3}}{2} z^{2}+\left(C_{3} f_{2}-C_{1} f_{3}\right) z+C_{5},
\end{gathered}
$$

здесь $C_{1}, C_{2}, C_{3}, C_{4}, C_{5}$ - постоянные интегрирования.

1.2. Если $u_{1}>0$, то гидродинамические поля описываются тригонометрическими и квазиполиномиальными выражениями:

1.3 .

$$
\begin{gathered}
V=\frac{\alpha_{2} z+p_{2}}{u_{1}}+C_{1} \sin (k z)+C_{2} \cos (k z) ; \\
U=-\frac{\alpha_{2}\left(u_{2}-f_{1}\right)+\alpha_{1} u_{1}}{u_{1}^{2}} z-\frac{p_{2}\left(u_{2}-f_{1}\right)+p_{1} u_{1}}{u_{1}^{2}}-\frac{C_{1}\left(u_{2}-f_{1}\right)}{2 u_{1}} \sin (k z)-\frac{C_{2}\left(u_{2}-f_{1}\right)}{2 u_{1}} \cos (k z)+ \\
+C_{3} \exp (-k z)+C_{3} \exp (k z) ;
\end{gathered}
$$




$$
\begin{gathered}
P_{0}=-\frac{f_{3} \alpha_{2} u_{1}+f_{2}\left(\left(u_{2}-f_{1}\right) \alpha_{2}+\alpha_{1} u_{1}\right)}{2 u_{1}^{2}} z^{2}-\frac{f_{3} p_{2} u_{1}+f_{2}\left(\left(u_{2}-f_{1}\right) p_{2}+p_{1} u_{1}\right)}{u_{1}^{2}} z+C_{5}- \\
-\frac{C_{2}\left(2 f_{3} u_{1}+f_{2}\left(u_{2}-f_{1}\right)\right)}{2 k u_{1}} \sin (k z)+\frac{C_{1}\left(2 f_{3} u_{1}+f_{2}\left(u_{2}-f_{1}\right)\right)}{2 k u_{1}} \cos (k z)- \\
-\frac{C_{3} f_{2}}{k} \exp (-k z)+\frac{C_{4} f_{2}}{k} \exp (k z) .
\end{gathered}
$$

Здесь введено обозначение для параметра $k=\sqrt{\frac{u_{1}}{v}}$.

1.4. Если $u_{1}<0$, то получим следующий вид фоновых скоростей и фонового давления [54]:

$$
\begin{gathered}
V=\frac{\alpha_{2} z+p_{2}}{u_{1}}+C_{1} \sinh (k z)+C_{2} \cosh (k z) ; \\
U=-\frac{\left(u_{2}-f_{1}\right) \alpha_{2}+\alpha_{1} u_{1}}{u_{1}^{2}} z-\frac{\left(u_{2}-f_{1}\right) p_{2}+p_{1} u_{1}}{u_{1}^{2}}-\frac{C_{1}\left(u_{2}-f_{1}\right)}{2 u_{1}} \sinh (k z)-\frac{C_{2}\left(u_{2}-f_{1}\right)}{2 u_{1}} \cosh (k z)+ \\
+C_{3} \sin (k z)+C_{3} \cos (k z) ; \\
P_{0}=-\frac{f_{3} \alpha_{2} u_{1}+f_{2}\left(\left(u_{2}-f_{1}\right) \alpha_{2}+\alpha_{1} u_{1}\right)}{2 u_{1}^{2}} z^{2}-\frac{f_{3} p_{2} u_{1}+f_{2}\left(\left(u_{2}-f_{1}\right) p_{2}+u_{1} p_{1}\right)}{u_{1}^{2}} z+C_{5}- \\
-\frac{C_{2}\left(2 f_{3} u_{1}+f_{2}\left(u_{2}-f_{1}\right)\right)}{2 k u_{1}} \sinh (k z)-\frac{C_{1}\left(2 f_{3} u_{1}+f_{2}\left(u_{2}-f_{1}\right)\right)}{2 k u_{1}} \cosh (k z),
\end{gathered}
$$

где $k=\sqrt{\frac{\left(-u_{1}\right)}{v}}$.

2. Пусть теперь $v_{1}+f_{1} \neq 0$. В этом случае из первого уравнения системы (21) можем выразить скорость $U$ :

$$
U=\frac{v V^{\prime \prime}+u_{1} V-P_{2}}{v_{1}+f_{1}}
$$

В результате подстановки выражения (29) во второе уравнение подсистемы (21) получим следующее линейное дифференциальное уравнение четвертого порядка с постоянными коэффициентами для определения скорости $V$ :

$$
V^{(4)}+S \cdot V=\frac{P_{1}\left(v_{1}+f_{1}\right)-u_{1} P_{2}}{v^{2}},
$$

где $S=-\frac{u_{1}^{2}+\left(u_{2}-f_{1}\right)\left(v_{1}+f_{1}\right)}{v^{2}}$. Величина коэффициента $S$ влияет на структуру решения неоднородного уравнения (30). 
2.1. Если $S=0$, то получим представление компонент поля скорости и поля давления:

$$
\begin{aligned}
& V=\frac{\alpha_{1}\left(v_{1}+f_{1}\right)-u_{1} \alpha_{2}}{120 v^{2}} z^{5}+\frac{p_{1}\left(v_{1}+f_{1}\right)-u_{1} p_{2}}{24 v^{2}} z^{4}+C_{4} z^{3}+C_{3} z^{2}+C_{2} z+C_{1} ; \\
& U=\frac{u_{1}\left(\alpha_{1}\left(f_{1}+v_{1}\right)-\alpha_{2} u_{1}\right)}{120 v^{2}\left(f_{1}+v_{1}\right)} z^{5}+\frac{u_{1}\left(p_{1}\left(f_{1}+v_{1}\right)-p_{2} u_{1}\right)}{24 v^{2}\left(f_{1}+v_{1}\right)} z^{4}+\frac{6 v C_{4} u_{1}+\alpha_{1}\left(f_{1}+v_{1}\right)-\alpha_{2} u_{1}}{6 v\left(f_{1}+v_{1}\right)} z^{3}+ \\
& +\frac{2 v C_{3} u_{1}+p_{1}\left(f_{1}+v_{1}\right)-p_{2} u_{1}}{2 v\left(f_{1}+v_{1}\right)} z^{\wedge} 2+\frac{\left(6 v C_{4}+C_{2} u_{1}-\alpha_{2}\right)}{f_{1}+v_{1}} z+\frac{2 v C_{3}-p_{2}+C_{1} u_{1}}{f_{1}+v_{1}} ; \\
& P_{0}=-\frac{\left(f_{1} f_{3}-f_{2} u_{1}+f_{3} v_{1}\right)\left(f_{1} \alpha_{1}+v_{1} \alpha_{1}-u_{1} \alpha_{2}\right)}{720 v^{2}\left(f_{1}+v_{1}\right)} z^{6}-\frac{\left(f_{1} f_{3}-f_{2} u_{1}+f_{3} v_{1}\right)\left(f_{1} p_{1}-p_{2} u_{1}+p_{1} v_{1}\right)}{120 v^{2}\left(f_{1}+v_{1}\right)} z^{5}+ \\
& +\frac{-6 v C_{4}\left(f_{1} f_{3}-f_{2} u_{1}+f_{3} v_{1}\right)+f_{2}\left(f_{1} \alpha_{1}+v_{1} \alpha_{1}-u_{1} \alpha_{2}\right)}{24 v\left(f_{1}+v_{1}\right)} z^{4}+ \\
& +\frac{-2 v C_{3}\left(f_{1} f_{3}-f_{2} u_{1}+f_{3} v_{1}\right)+f_{2}\left(f_{1} p_{1}-p_{2} u_{1}+p_{1} v_{1}\right)}{6 v\left(f_{1}+v_{1}\right)} z^{3}- \\
& -\frac{-6 v C_{4} f_{2}+C_{2}\left(f_{1} f_{3}-f_{2} u_{1}+f_{3} v_{1}\right)+f_{2} \alpha_{2}}{2\left(f_{1}+v_{1}\right)} z^{2}-\frac{-2 v C_{3} f_{2}+f_{2} p_{2}+C_{1}\left(f_{1} f_{3}-f_{2} u_{1}+f_{3} v_{1}\right)}{f_{1}+v_{1}} z+C_{5},
\end{aligned}
$$

Здесь $C_{1} ; C_{2} ; C_{3} ; C_{4} ; C_{5}$ - постоянные интегрирования). ния [54]:

2.2. Если $S>0$, то имеем другое точное решение для описания скоростей и давле-

$$
\begin{aligned}
& V=C_{1} \cosh (k z) \cos (k z)+C_{2} \cosh (k z) \sin (k z)+C_{3} \sinh (k z) \cos (k z)+C_{4} \sinh (k z) \sin (k z)+ \\
& +\frac{\alpha_{1}\left(v_{1}+f_{1}\right)-u_{1} \alpha_{2}}{S v^{2}} z+\frac{p_{1}\left(v_{1}+f_{1}\right)-u_{1} p_{2}}{S v^{2}} ; \\
& U=\frac{\left(2 k^{2} v C_{4}+C_{1} u_{1}\right)}{f_{1}+v_{1}} \cosh (k z) \cos (k z)+\frac{\left(-2 k^{2} v C_{3}+C_{2} u_{1}\right)}{f_{1}+v_{1}} \cosh (k z) \sin (k z)+ \\
& +\frac{\left(2 k^{2} v C_{2}+C_{3} u_{1}\right)}{f_{1}+v_{1}} \sinh (k z) \cos (k z)+\frac{\left(-2 k^{2} v C_{1}+C_{4} u_{1}\right)}{f_{1}+v_{1}} \sinh (k z) \sin (k z)+ \\
& +\frac{f_{1} u_{1} \alpha_{1}+u_{1} v_{1} \alpha_{1}-S v^{2} \alpha_{2}-u_{1}^{2} \alpha_{2}}{S v^{2}\left(f_{1}+v_{1}\right)} z+\frac{-S v^{2} p_{2}+f_{1} p_{1} u_{1}-p_{2} u_{1}^{2}+p_{1} u_{1} v_{1}}{S v^{2}\left(f_{1}+v_{1}\right)} ; \\
& P_{0}=\frac{C_{3}\left(f_{2}\left(2 k^{2} v+u_{1}\right)-f_{3}\left(f_{1}+v_{1}\right)\right)+C_{2}\left(f_{2}\left(2 k^{2} v-u_{1}\right)+f_{3}\left(f_{1}+v_{1}\right)\right)}{2 k\left(f_{1}+v_{1}\right)} \cosh (k z) \cos (k z)+
\end{aligned}
$$




$$
\begin{aligned}
& +\frac{C_{4}\left(f_{2}\left(2 k^{2} v+u_{1}\right)-f_{3}\left(f_{1}+v_{1}\right)\right)-C_{1}\left(f_{2}\left(2 k^{2} v-u_{1}\right)+f_{3}\left(f_{1}+v_{1}\right)\right)}{2 k\left(f_{1}+v_{1}\right)} \cosh (k z) \sin (k z)+ \\
& +\frac{C_{1}\left(f_{2}\left(2 k^{2} v+u_{1}\right)-f_{3}\left(f_{1}+v_{1}\right)\right)+C_{4}\left(f_{2}\left(2 k^{2} v-u_{1}\right)+f_{3}\left(f_{1}+v_{1}\right)\right)}{2 k\left(f_{1}+v_{1}\right)} \sinh (k z) \cos (k z)+ \\
& +\frac{C_{2}\left(f_{2}\left(2 k^{2} v+u_{1}\right)-f_{3}\left(f_{1}+v_{1}\right)\right)-C_{3}\left(f_{2}\left(2 k^{2} v-u_{1}\right)+f_{3}\left(f_{1}+v_{1}\right)\right)}{2 k\left(f_{1}+v_{1}\right)} \sinh (k z) \sin (k z)+ \\
& +\frac{-\left(f_{1}+v_{1}\right)\left(-f_{2} u_{1}+f_{3}\left(f_{1}+v_{1}\right)\right) \alpha_{1}+\left(-f_{2}\left(S v^{2}+u_{1}^{2}\right)+f_{3} u_{1}\left(f_{1}+v_{1}\right)\right) \alpha_{2}}{2 S v^{2}\left(f_{1}+v_{1}\right)} z^{2}- \\
& -\frac{S v^{2} f_{2} p_{2}+\left(-f_{2} u_{1}+f_{3}\left(f_{1}+v_{1}\right)\right)\left(-p_{2} u_{1}+p_{1}\left(f_{1}+v_{1}\right)\right)}{S v^{2}\left(f_{1}+v_{1}\right)}+C_{5} .
\end{aligned}
$$

здесь $k^{4}=S / 4$.

2.3. Если $S<0$, то в заключительном случае получим следующие формулы для вычисления компонент скоростей и давления [54]:

$$
\begin{aligned}
& V=C_{1} \cos (k z)+C_{2} \sin (k z)+C_{3} \cosh (k z)+C_{4} \sinh (k z)+ \\
& +\frac{\alpha_{1}\left(v_{1}+f_{1}\right)-u_{1} \alpha_{2}}{S v^{2}} z+\frac{p_{1}\left(v_{1}+f_{1}\right)-u_{1} p_{2}}{S v^{2}} ; \\
& U=\frac{C_{1}\left(u_{1}-k^{2} v\right)}{f_{1}+v_{1}} \cos (k z)+\frac{C_{2}\left(u_{1}-k^{2} v\right)}{f_{1}+v_{1}} \sin (k z)+\frac{C_{3}\left(u_{1}+k^{2} v\right)}{f_{1}+v_{1}} \cosh (k z)+\frac{C_{4}\left(u_{1}+k^{2} v\right)}{f_{1}+v_{1}} \sinh (k z)+ \\
& +\frac{u_{1}\left(\left(f_{1}+v_{1}\right) \alpha_{1}-u_{1} \alpha_{2}\right)-S v^{2} \alpha_{2}}{S v^{2}\left(f_{1}+v_{1}\right)} z+\frac{u_{1}\left(-p_{2} u_{1}+p_{1}\left(f_{1}+v_{1}\right)\right)-S v^{2} p_{2}}{S v^{2}\left(f_{1}+v_{1}\right)} ; \\
& P_{0}=\frac{C_{4}\left(f_{2}\left(k^{2} v+u_{1}\right)-f_{3}\left(f_{1}+v_{1}\right)\right)}{k\left(f_{1}+v_{1}\right)} \cosh (k z)+\frac{C_{3}\left(f_{2}\left(k^{2} v+u_{1}\right)-f_{3}\left(f_{1}+v_{1}\right)\right)}{k\left(f_{1}+v_{1}\right)} \sinh (k z)+ \\
& +\frac{C_{2}\left(f_{2}\left(k^{2} v-u_{1}\right)+f_{3}\left(f_{1}+v_{1}\right)\right)}{k\left(f_{1}+v_{1}\right)} \cos (k z)-\frac{C_{1}\left(f_{2}\left(k^{2} v-u_{1}\right)+f_{3}\left(f_{1}+v_{1}\right)\right)}{k\left(f_{1}+v_{1}\right)} \sin (k z)+ \\
& +\frac{-\left(f_{1}+v_{1}\right)\left(-f_{2} u_{1}+f_{3}\left(f_{1}+v_{1}\right)\right) \alpha_{1}+\left(-f_{2}\left(S v^{2}+u_{1}^{2}\right)+f_{3} u_{1}\left(f_{1}+v_{1}\right)\right) \alpha_{2}}{2 S v^{2}\left(f_{1}+v_{1}\right)} z^{2}-
\end{aligned}
$$




$$
-\frac{S v^{2} f_{2} p_{2}+\left(-f_{2} u_{1}+f_{3}\left(f_{1}+v_{1}\right)\right)\left(-p_{2} u_{1}+p_{1}\left(f_{1}+v_{1}\right)\right)}{S v^{2}\left(f_{1}+v_{1}\right)} z+C_{5}
$$

Таким образом, если существует вещественный корень $c$ уравнения (25), то переопределенная система (19) имеет совместное решение (26). На основании решения (26), согласно формулам (27), определяется вид градиентов давления $P_{1}$ и $P_{2}$. С помощью этих выражений и решения для пространственных ускорений (26) находятся однородные скорости $U$ и $V$ и фоновое давление $P_{0}$, которые в зависимости от значений параметров задачи определяются формулами, приведенными в рамках случаев 1.1-2.3. Набор указанных функций есть совместное точное (аналитическое) решение переопределенной нелинейной системы дифференциальных уравнений (18)-(22).

Заметим, что для сдвиговых течений третий параметр Кориолиса $f_{3}$ играет роль только при определении структуры фонового давления $P_{0}$. Если положить $f_{3}=0$, придем к случаю двух параметров Кориолиса, подробно исследованному в [26]. Случай одного параметра Кориолиса [25] получается, если дополнительно приравнять нулю и величину $f_{2}$. Таким образом, построенное в данной статье решение обобщает ранее полученные результаты.

\section{4. Заключение}

В статье получено условие совместности редуцированной системы, описывающей неоднородное сдвиговое течение вращающейся вязкой изотермической жидкости в случае, когда сила инерции в уравнениях Навье-Стокса характеризуется тремя параметрами Кориолиса. Показано, что пространственные ускорения поля скорости при этом являются постоянными функциями. Также построено точное решение для поля давления и однородных компонент поля скорости. Приведенное решение является обобщением исследований по анализу переопределенных систем, описывающих изотермическое вращение жидкости в случаях одного и двух ненулевых параметров Кориолиса.

\section{Литература}

1. Монин А. С. Теоретические основы геофизической гидродинамики. - Л. : Гидрометеоиздат, 1988.

2. Бреховских Л. М., Гончаров В. В. Введение в механику сплошных сред (в приложении к теории волн) / отв. ред. Г. И. Баренблатт. - М. : Наука, 1982.

3. Зырянов В. Н. Теория установившихся океанических течений. - Ленинград : Гидрометеоиздат, 1985.

4. Педлоски Дж. Геофизическая гидродинамика : в 2 т. - М. : Мир, 1984.

5. $\quad$ Гилл А. Динамика атмосферы и океана. - М. : Мир, 1986.

6. Ekman V. W. On the Influence of the Earth's Rotation on Ocean-Currents // Ark. Mat. Astron. Fys. - 1905. - Vol. 2, no. 11. - P. 1-52.

7. Гершуни Г. З., Жуховицкий Е. М. Конвективная неустойчивость несжимаемой жидкости. - М. : Наука, 1972.

8. Ландау Л. Д., Лифшиц Е. М. Гидродинамика. - 6-е. изд. - М. : Физматлит, 2006.

9. Темам Р. Уравнения Навье-Стокса. Теория и численный анализ. - М. : Изд-во «Мир», 1981.

10. Химическая гидродинамика / А. М. Кутепов, А. Д. Полянин, 3. Д. Запрянов, А. В. Вязьмин, Д. А. Казенин. - М. : Квантум, 1996. 
11. Couette M. Etudes sur le frottement des liquids // Ann. Chim. Phys. - 1890. - Vol. 21. P. 433-510.

12. Start-up flows in a three-dimensional rectangular driven cavity of aspect ratio 1:1:2 at $\operatorname{Re}=1000$ / J. L. Guermond, C. Migeon, G. Pineau, L. Quartapel // J. Fluid Mech. - 2002. Vol. 450. - P. 169-199. - DOI: 10.1017/S0022112001006383.

13. Boundary slip in Newtonian liquids: a review of experimental studies / C. Neto, D. Evans, E. Bonaccurso, H.-J. Butt, V.S.J. Craig // Reports on Progress in Physics. - 2005. - Vol. 68. P. 2859-2897. - DOI: 10.1088/0034-4885/68/12/R05.

14. Aristov S. N., Frik P. G. Nonlinear effects of the Ekman layer on the dynamics of largescale eddies in shallow water // Journal of Applied Mechanics and Technical Physics. - 1991. Vol. 32, no. 2. - P. 189-194. - DOI: 10.1007/BF00858033.

15. Аристов С. Н., Шварц К. Г. Вихревые течения адвективной природы во вращающемся слое жидкости. - Пермь : Изд-во Пермск. гос. ун-та, 2006.

16. Аристов С. Н., Шварц К. Г. Вихревые течения в тонких слоях жидкости. - Киров : ВятГУ, 2011.

17. Горшков А. В., Просвиряков Е. Ю. Конвективное слоистое течение Экмана вязкой несжимаемой жидкости // Известия РАН. Физика атмосферы и океана. - 2018. - Т. 54, вып. 2. C. 213-220. - DOI: 10.7868/S0003351518020101.

18. Компаниец Л. А., Питальская О. С. Точные решения модели Экмана трехмерного ветрового движения однородной жидкости с учетом геострофической составляющей // Компьютерные исследования и моделирование. - 2009. - Т. 1, вып. 1. - С. 57-66. - DOI: 10.20537/2076-7633-20091-1-57-66.

19. Аристов С. Н., Князев Д. В., Полянин А. Д. Точные решения уравнений Навье-Стокса с линейной зависимостью компонент скорости от двух пространственный переменных // Теоретические основы химической технологии. - 2009. - Т. 43, вып. 5. - С. 547-566.

20. Shrira V. I., Almelah R. B. Upper-ocean Ekman current dynamics: a new perspective // Journal of Fluid Mechanics. - 2020. - Vol. 887. - A24. - DOI: 10.1017/jfm.2019.1059.

21. Existence and uniqueness and first order approximation of solutions to atmospheric Ekman flows / M. Fečkan, Y. Guan, D. O’Regan, J. R. Wang // Monatshefte für Mathematik. - 2020. DOI: $10.1007 / \mathrm{s} 00605-020-01414-7$.

22. Global modes and large-scale structures in an Ekman boundary layer / J. L. Ortiz-Tarin, S. Lee, O. Flores, S. Sarkar // Journal of Physics: Conference Series. - 2020. - Vol. 1522. -012011. DOI:10.1088/1742-6596/1522/1/012011.

23. Constantin A., Johnson R. S. Atmospheric Ekman flows with variable eddy viscosity // Boundary-Layer Meteorol. - 2019. - Vol. 170. - P. 395-414. - DOI: 10.1007/s 10546-018-0404-0.

24. Просвиряков Е. Ю. Новый класс точных решений уравнений Навье-Стокса со степенной зависимостью скоростей от двух пространственных координат // Теоретические основы химической технологии. - 2019. - Т. 53, вып. 1. - С. 112-120. - DOI: 10.1134/S0040357118060118.

25. Бурмашева Н. В., Просвиряков Е. Ю. Точное решение уравнений Навье-Стокса, описывающее пространственно неоднородные течения вращающейся жидкости // Труды Института математики и механики УрО РАН. - 2020. - Т. 26, вып. 2. - С. 79-87.

26. Бурмашева Н. В., Просвиряков Е. Ю. Класс точных решений для двумерных уравнений геофизической гидродинамики с двумя параметрами Кориолиса // Известия Иркутского государственного университета. Серия Математика. - 2020. - Т. 32. - С. 33-48. DOI: $10.26516 / 1997-7670.2020 .32 .33$.

27. Lin C. C. Note on a class of exact solutions in magneto-hydrodynamics // Arch. Rational Mech. Anal. - 1958. - Vol. 1. - P. 391-395.

28. Аристов С. Н., Просвиряков Е. Ю. Крупномасштабные течения завихренной вязкой несжимаемой жидкости // Известия высших учебных заведений. Авиационная техника. 2015. - Вып. 4. - С. 50-54. 
29. Аристов С. Н., Просвиряков Е. Ю. Неоднородные течения Куэтта // Нелинейная динамика. - 2014. - Т. 10, вып. 2. - C. 177-182. - DOI: 10.20537/nd1402004.

30. Просвиряков Е. Ю. Точные решения трехмерных потенциальных и завихренных течений Куэтта вязкой несжимаемой жидкости // Вестник Национального исследовательского ядерного университета МИФИ. - 2015. - Т. 4, вып. 6. - С. 501-506. - DOI: 10.1134/S2304487X15060127.

31. Аристов С. Н., Просвиряков Е. Ю. Нестационарные слоистые течения завихренной жидкости // Известия Российской Академии Наук. Механика жидкости и газа. - 2016. Вып. 2. - С. 25-31.

32. Аристов С. Н., Просвиряков Е. Ю. Новый класс точных решений трехмерных уравнений термодиффузии // Теоретические основы химической технологии. $-2016 .-$ Т. 50, вып. 3. C. 294-301. - DOI: 10.7868/S0040357116030027.

33. Бурмашева Н. В., Просвиряков Е. Ю. Крупномасштабная слоистая стационарная конвекция вязкой несжимаемой жидкости под действием касательных напряжений на верхней границе. Исследование поля скоростей // Вестн. Сам. гос. техн. ун-та. Сер. Физ.-мат. науки. 2017. - Т. 21, вып. 1. - C. 180-196. - DOI: 10.14498/vsgtu1527.

34. Бурмашева Н. В., Просвиряков Е. Ю. Крупномасштабная слоистая стационарная конвекция вязкой несжимаемой жидкости под действием касательных напряжений на верхней границе. Исследование полей температуры и давления // Вестн. Сам. гос. техн. ун-та. Cер. Физ.-мат. науки. - 2017. - Т. 21, вып. 4. - C. 736-751. - DOI: 10.14498/vsgtu1568.

35. Горшков А. В., Просвиряков Е. Ю. Аналитические решения стационарной сложной конвекции, описывающие поле касательных напряжений разного знака // Тр. ИММ УpO РАН. - 2017. - Т. 23, вып. 2. - С. 32-41. - DOI: 10.21538/0134-4889-2017-23-2-32-41.

36. Верещагин В. П., Субботин Ю. Н., Черных Н. И. Описание винтового движения несжимаемой невязкой жидкости // Тр. ИММ УрО РАН. - 2014. - Т. 20, вып. 1. - С. 43-51.

37. Верещагин В. П., Субботин Ю. Н., Черных Н. И. Некоторые решения уравнений движения для несжимаемой вязкой сплошной среды // Тр. ИММ УрО РАН. - 2013. - Т. 19, вып. 4. - С. 48-63.

38. Верещагин В. П., Субботин Ю. Н., Черных Н. И. К механике винтовых потоков в идеальной несжимаемой невязкой сплошной среде // Тр. ИММ УрО РАН. - 2012. - Т. 18, вып. 4. С. $120-134$.

39. Зубарев Н. М., Просвиряков Е. Ю. О точных решениях для слоистых трехмерных нестационарных изобарических течений вязкой несжимаемой жидкости // Прикладная механика и техническая физика. - 2019. - Т. 60, вып. 6(358). - С. 65-71. - DOI: 10.15372/PMTF20190607.

40. Пухначев В. В. Точечный вихрь в вязкой несжимаемой жидкости // Прикладная механика и техническая физика. - 2014. - Т. 55, вып. 2. - С. 180-187.

41. Бирих Р. В., Пухначев В. В., Фроловская О. А. Конвективное течение в горизонтальном канале с неньютоновской реологией поверхности при нестационарном продольном градиенте температуры // Механика жидкости и газа. - 2015. - Вып. 1. - С. 192-198.

42. Голубкин В. Н., Сизых Г. Б. Принцип максимума функции Бернулли // Ученые записки ЦАГИ. - 2015. - Т. 46, вып. 5. - С. 43-46.

43. Сизых Г.Б. Осесимметричные винтовые течения вязкой жидкости // Изв. вузов. Матем. - 2019. - Вып. 2. - С. 49-56.

44. Сизых Г. Б. Расщепление уравнений Навье-Стокса для одного класса осесимметричных течений // Вестн. Сам. гос. техн. ун-та. Сер. Физ.-мат. науки. - 2020. - Т. 24, вып. 1. C. 163-173. - DOI: 10.14498/vsgtu1740.

45. Марков В. В., Сизых Г. Б. Точные решения уравнений Эйлера для некоторых двумерных течений несжимаемой жидкости // Тр. МИАН. - 2016. - Т. 294. - С. 300-307. DOI: $10.1134 / \mathrm{S} 0371968516030195$. 
46. Ershkov S. V., Shamin R. V., Giniyatullina A. R. On a new type of non-stationary helical flows for incompressible 3D Navier-Stokes equations // Journal of King Saud University - Science. 2020. - Vol. 32, no. 1. - P. 459-467. - DOI: 10.1016/j.jksus.2018.07.006.

47. Ковалёв В. П., Просвиряков Е. Ю., Сизых Г. Б. Получение примеров точных решений уравнений Навье-Стокса для винтовых течений методом суммирования скоростей // Труды Московского физико-технического института. - 2017. - Т. 9, вып. 1 (33). - С. 71-88.

48. Бурмашева Н. В., Просвиряков Е. Ю. Термокапиллярная конвекция вертикально завихренной жидкости // Теоретические основы химической технологии. - 2020. - Т. 54, вып. 1. - C. 114-124. - DOI: 10.31857/S0040357119060034.

49. Ульянов О. Н. Об одном классе течений вязкой жидкости // Тр. ИММ УрО РАН. 2003. - Т. 9, вып. 2. - С. 129-136.

50. Pukhnachev V. V. Nonlinear diffusion and exact solutions to the Navier-Stokes equations // The Bulletin of Irkutsk State University. Series Mathematics. - 2010. - Vol 3, no.1. - P. 61-69.

51. Burmasheva N. V., Prosviryakov E. Yu. Convective layered flows of a vertically whirling viscous incompressible fluid. Velocity field investigation // Вестник СамГТУ. Сер. Физ.-мат. науки. - 2019. - Т. 23, вып. 2. - C. 341-360. - DOI: 10.14498/vsgtu1670.

52. Privalova V. V., Prosviryakov E. Yu., Simonov M. A. Nonlinear gradient flow of a vertical vortex fl uid in a thin layer // Нелинейн. динам. - 2019. - Т. 15, вып. 3. - С. 271-283. DOI: $10.20537 / \mathrm{nd} 190306$.

53. Аристов С. Н., Привалова В. В., Просвиряков Е. Ю. Стационарное неизотермическое течение Куэтта. Квадратичный нагрев верхней границы слоя жидкости // Нелинейная динам. 2016. - Т. 12, вып. 2. - С. 167-178.

54. Polyanin A. D., Zaitsev V. F. Handbook of exact solutions for ordinary differential equations. - 2nd ed. - Boca Raton : Chapman\& Hall/CRC, 2003. 